\title{
水热法合成 $\mathrm{Bi}_{2} \mathrm{WO}_{6} / \mathrm{CNOs}$ 纳米材料及其电化学性能
}

\author{
王佳玮，杨艳青，高泽宇，梁颖，邓钏，张卫珂 \\ (太原理工大学 环境科学与工程学院, 太原 030024)
}

摘 要: 为了提高超级电容器的性能, 尽可能减少环境污染, 电极材料尤其是低成本、高能量密度的环保型电极材 料受到人们越来越多的关注。本研究采用水热法制备了 $\mathrm{Bi}_{2} \mathrm{WO}_{6} / \mathrm{CNOs}(\mathrm{CNOs}$, 纳米洋葱碳 $)$ 和 $\mathrm{Bi}_{2} \mathrm{WO}_{6}$ 纳米材料。 采用扫描电子显微镜(SEM)和 X 射线晶体衍射(XRD)对产物的形貌、结构进行分析, 并通过循环伏安法、恒电流充 放电测试、交流阻抗对材料的电化学性能进行评价。结果表明, 以 $1 \mathrm{~mol} \cdot \mathrm{L}^{-1} \mathrm{KOH}$ 为电解液, 电流密度为 $2 \mathrm{~mA} \cdot \mathrm{cm}^{-2}$ 时, $\mathrm{Bi}_{2} \mathrm{WO}_{6} / \mathrm{CNOs}$ 与纯相 $\mathrm{Bi}_{2} \mathrm{WO}_{6}$ 的比电容分别为 328 和 $218 \mathrm{~F} \cdot \mathrm{g}^{-1}$; 电流密度为 $5 \mathrm{~mA} \cdot \mathrm{cm}^{-2}$ 时, 经过 300 次循环, $\mathrm{Bi}_{2} \mathrm{WO}_{6} / \mathrm{CNOs}$ 的比容量保持率比纯相 $\mathrm{Bi}_{2} \mathrm{WO}_{6}$ 提高了 $34.37 \%$ 。可见, 在 $\mathrm{Bi}_{2} \mathrm{WO}_{6}$ 中加入 $\mathrm{CNOs}$ 能明显改善 $\mathrm{Bi}_{2} \mathrm{WO}_{6}$ 的电化学性能。

关 键 词: 铇酸铋; 纳米洋葱碳; 电化学性能; 超级电容器

中图分类号: TQ127 文献标识码: A

\section{Electrochemical Performance of $\mathrm{Bi}_{2} \mathrm{WO}_{6} / \mathrm{CNOs}$ Nanocomposites Synthesized via a Hydrothermal Method}

\author{
WANG Jia-Wei, YANG Yan-Qing, GAO Ze-Yu, LIANG Ying, DENG Chuan, ZHANG Wei-Ke \\ (College of Environmental Science and Engineering, Taiyuan University of Technology, Taiyuan 030024, China)
}

\begin{abstract}
In order to enhance the performance of supercapacitor and reduce environmental pollution as far as possible, electrode materials especially low-cost and eco-friendly electrode materials with high energy density have attracted a great deal of attention. In this paper, $\mathrm{Bi}_{2} \mathrm{WO}_{6} / \mathrm{CNOs}$ (CNOs, Carbon Nano Onions) and $\mathrm{Bi}_{2} \mathrm{WO}_{6}$ nanocomposites have been synthesized via a hydrothermal method. And the structures and morphologies of samples were characterized by X-ray diffraction (XRD) and scanning electron microscopy (SEM). The electrochemical performances of the as-prepared samples were investigated by cyclic voltammetry, galvanostatic charge - discharge measurements and electrochemical impedance spectroscopy. At a current density of $2 \mathrm{~mA} \cdot \mathrm{cm}^{-2}$, the specific capacitance of the $\mathrm{Bi}_{2} \mathrm{WO}_{6} / \mathrm{CN}-$ Os and $\mathrm{Bi}_{2} \mathrm{WO}_{6}$ was $328 \mathrm{~F} \cdot \mathrm{g}^{-1}$ and $218 \mathrm{~F} \cdot \mathrm{g}^{-1}$ with $1 \mathrm{~mol} \cdot \mathrm{L}^{-1} \mathrm{KOH}$ served as electrolyte, respectively. After 300 charge-discharge cycles at a current density of $5 \mathrm{~mA} \cdot \mathrm{cm}^{-2}$, the specific capacitance of the $\mathrm{Bi}_{2} \mathrm{WO}_{6} / \mathrm{CNO}$ improved $34.37 \%$ compared with pure $\mathrm{Bi}_{2} \mathrm{WO}_{6}$. Thus, CNOs can enhance the electrochemical performance of $\mathrm{Bi}_{2} \mathrm{WO}_{6}$ obvously.
\end{abstract}

Key words: $\mathrm{Bi}_{2} \mathrm{WO}_{6}$; carbon nano onions; electrochemical analysis; supercapacitors

收稿日期: 2018-03-14; 收到修改稿日期：2018-04-15

基金项目: 中国博士后第 59 批面上基金(2016M592654); 山西省基础研究计划项目(2015021062); 山西省高等学校科技创 新研究项目(STIP, 2016147)

The 59th Batch of Open Category China Postdoctoral Funds (2016M592654); Natural Science Foundation of Shanxi Province (2015021062); Scientific and Technological Innovation Programs of Higher Education Institutions in Shanxi (STIP, 2016147)

作者简介：王佳玮(1993-), 女，硕士研究生.E-mail: 812308622@qq.com

通讯作者：张卫珂，副教授. E-mail: zhangweike@tyut.edu.cn 
近年来, 超级电容器作为一种多功能储能系统, 在能量和功率密度方面填补了电池和传统电容器的 空白, 备受人们关注 ${ }^{[1]}$ 。根据能量的储存原理, 超级 电容器可以分为双电层电容器和噟电容器。双电层 电容是在物理电场的作用下, 电荷在电极和电解质 界面积累而产生 ${ }^{[2]}$, 噟电容则主要发生在电极材料 表面, 与氧化还原反应产生的法拉第电子一电荷转 移的实现有关 ${ }^{[3]}$ 。当前, 超级电容器的研究主要集中 于在不影响功率密度的前提下开发具有高能量密 度、低成本和环保型的电极材料。

目前，对金属氧化物如 $\mathrm{NiO}\left(674.2 \mathrm{~F} \cdot \mathrm{g}^{-1}\right) 、 \mathrm{Co}_{3} \mathrm{O}_{4}$ $\left(905 \mathrm{~F} \cdot \mathrm{g}^{-1}\right) 、 \mathrm{MnO}_{2}\left(622 \mathrm{~F} \cdot \mathrm{g}^{-1}\right)$ 和 $\mathrm{NiWO}_{4}\left(173 \mathrm{~F} \cdot \mathrm{g}^{-1}\right)$ 等 ${ }^{[4-8]}$ 作为阳极材料已经进行了大量的研究, 对 $\mathrm{Bi}_{2} \mathrm{O}_{3}\left(386 \mathrm{~F} \cdot \mathrm{g}^{-1}\right) 、 \mathrm{Fe}_{3} \mathrm{O}_{4}\left(163 \mathrm{~F} \cdot \mathrm{g}^{-1}\right) 、 \mathrm{VN}\left(413 \mathrm{~F} \cdot \mathrm{g}^{-1}\right)$ 和 $\mathrm{BiPO}_{4}\left(302 \mathrm{~F} \cdot \mathrm{g}^{-1}\right)$ 等 ${ }^{[9-12]}$ 作为阴极材料的研究也 有一定进展, 然而上述材料用作活性材料产生的比 电容并不能满足实际的生产需求, 需要进一步改 进。据报道, $\mathrm{Bi}_{2} \mathrm{WO}_{6}$ 作为噟电容器的电极材料能够 提高电容器的比电容, Nithya 等 ${ }^{[13]}$ 以 $1 \mathrm{~mol} \cdot \mathrm{L}^{-1} \mathrm{KOH}$ 为电解液, 在电流密度为 $3 \mathrm{~mA} \cdot \mathrm{cm}^{-2}$ 的条件下, 将 $\mathrm{Bi}_{2} \mathrm{WO}_{6}$ 作为电容器的电极材料, 其比电容可达 $304 \mathrm{~F} \cdot \mathrm{g}^{-1}$, 经过 100 次循环 $\mathrm{Bi}_{2} \mathrm{WO}_{6}$ 的比电容保持率 为 $89 \%$, 这为提高电容器的性能提供了一个良好的 思路。然而, 电化学反应一般仅发生在噟电容材料 的表面, 只有一层非常薄的材料参与了电荷的存储 过程, 这限制了电化学性能的进一步提高。

迄今为止, 用来提高电极材料电化学性能的方 法主要包括(i)合成一维或二维的纳米结构; (ii)合成 金属氧化物/碳或三元复合材料等; (iii) 以一维或二 维的形态构造阵列; (iv)合成复杂异质结构的纳米材 料; (v)制备碳基复合材料作为活性物质; (vi)利用表 面活性剂/聚合物辅助合成纳米材料等 ${ }^{[14-17]}$ 。

其中, 碳基材料能够很大程度上提高其作为活 性材料的导电性, 降低电极材料的电阻率 ${ }^{[18-19]}$ 。纳 米洋葱碳(CNOs) 是 $\mathrm{Iijima}^{[20]}$ 在 1980 年发现的一种 新型碳材料, 具有结构均匀、直径小、导电率高和 易于分散等优点, 使其在储能方面得到越来越多的 重视。将 $\mathrm{CNOs}$ 和 $\mathrm{Bi}_{2} \mathrm{WO}_{6}$ 结合, 可以增加活性物质 $\mathrm{Bi}_{2} \mathrm{WO}_{6}$ 的导电性, 加快电子的转移速率, 并为电子 提供相对短的扩散路径, 从而加快活性物质与电解 液间的氧化还原反应，增加赝电容的比电容。

本文采用水热法合成了 $\mathrm{Bi}_{2} \mathrm{WO}_{6} / \mathrm{CNO}$ 和 $\mathrm{Bi}_{2} \mathrm{WO}_{6}$ 纳米材料, 并对材料的形貌、结构进行表征和分析, 通过循环伏安、恒流充放电和交流阻抗测试评价材 料的电化学性能。

\section{1 实验方法}

\section{1 $\mathrm{Bi}_{2} \mathrm{WO}_{6} 、 \mathrm{Bi}_{2} \mathrm{WO}_{6} / \mathrm{CNO}$ 的制备}

$1.455 \mathrm{~g}$ 硝酸铋(分析纯) (3 mmol)溶于 $6 \mathrm{~mL}$ $1.0 \mathrm{~mol} \cdot \mathrm{L}^{-1}$ 稀硝酸中, 标记为无色溶液 $\mathrm{A} 。 0.495 \mathrm{~g}$ 钨酸钠(分析纯) (1.5 mmol)溶于 $6 \mathrm{~mL}$ 去离子水中, 标记为无色溶液 $\mathrm{B}$ 。将溶液 $\mathrm{B}$ 逐滴加入 $\mathrm{A}$ 中, 生成 淡黄色悬浊液, 加入 $45 \mathrm{~mL}$ 乙二醇后调节 $\mathrm{pH}$ 至 $2 \sim 3$ 。 溶液搅拌 $30 \mathrm{~min}$ 后转入聚四氟乙烯反应釜中, 在 $150^{\circ} \mathrm{C}$ 加热 $2 \mathrm{~h}$ 。反应釜自然冷却到室温后, 用无水 乙醇和去离子水交替清洗沉淀 6 8 次, $80^{\circ} \mathrm{C}$ 干燥得 到 $\mathrm{Bi}_{2} \mathrm{WO}_{6}$ 样品。

$\mathrm{Bi}_{2} \mathrm{WO}_{6} / \mathrm{CNOs}$ 的合成只需将 $0.19 \mathrm{~g}$ 纯化后的 $\mathrm{CNOs}$ (购买自山西中兴环能科技有限公司)加入到 $45 \mathrm{~mL}$ 乙二醇中, 其余条件不变, 即可得到 $\mathrm{Bi}_{2} \mathrm{WO}_{6} /$ CNOs 纳米材料。

\section{2 材料的表征与测试}

用型号为 DX-2700X 的 $X$ 射线衍射分析仪 (XRD)对样品的组成和晶体结构进行分析; 用场发 射扫描电子显微镜(SEM) (JSM-6700)对材料的形貌 进行表征。

电化学性能分析在辰华 $760 \mathrm{E}$ 电化学工作站上进 行, 测试采用三电极体系: 铂电极为对电极, 饱和甘 录电极(SCE) 为参比电极, 工作电极的制备方法如下: 以制备好的材料为活性物质, 将质量比为 $8: 1: 1$ 的 活性材料、导电炭黑(Super-p)和聚偏氟乙烯(PVDF) 在乙醇中充分混合形成浆料, 均匀涂抹在 $1 \mathrm{~cm}^{2}$ 的 泡沫镍上, $60^{\circ} \mathrm{C}$ 下干燥 $24 \mathrm{~h}$ 。以 $1 \mathrm{~mol} \cdot \mathrm{L}^{-1}$ 的 $\mathrm{KOH}$ 溶 液为电解液, 对材料进行电化学性能分析。

\section{2 结果与讨论}

\subsection{SEM 和 XRD 分析}

图 1(a)为 CNOs 的 SEM 照片, 可以看出, 纯化 后的 CNOs 呈现出较为均匀的球状结构, 周围还有 部分碳纳米管和金属颗粒等杂质，粒径分布在 2 $120 \mathrm{~nm}$; 图 1(b) 是纯相 $\mathrm{Bi}_{2} \mathrm{WO}_{6}$ 的 SEM 照片, 纯相 $\mathrm{Bi}_{2} \mathrm{WO}_{6}$ 呈现出纳米片层状结构, 颗粒大小在 $100 \mathrm{~nm}$ 左右; 图 1(c)为 $\mathrm{Bi}_{2} \mathrm{WO}_{6} / \mathrm{CNO}$ 的 SEM 照片, $\mathrm{Bi}_{2} \mathrm{WO}_{6}$ 的片层状结构在与 CNOs 复合后变得粗粘且不规则, 表明 CNOs 对铇酸铋的形貌产生了较大的影响; 此 外, CNOs 分布在 $\mathrm{Bi}_{2} \mathrm{WO}_{6}$ 的周围, 这种分布可以达 到使二者紧密接触的目的, 由于 CNOs 具有良好的 导电性，因而可以使电子进入活性物质的速率加快, 从而改善 $\mathrm{Bi}_{2} \mathrm{WO}_{6} / \mathrm{CNO}$ 的电阻率。图 1(d)为 $\mathrm{CNOs}$ 、 

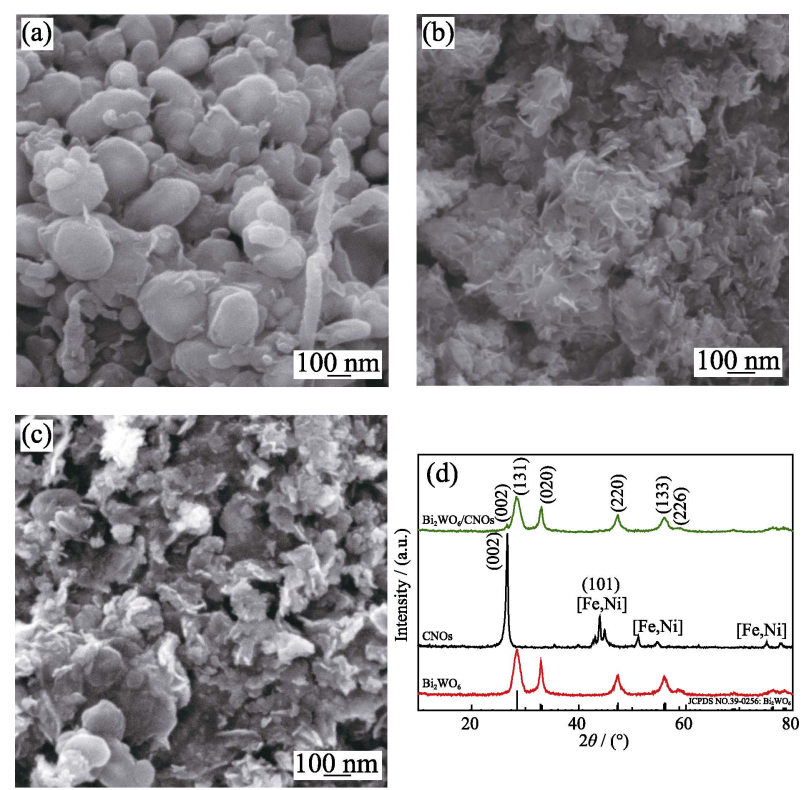

图 1 (a) CNOs、 (b) $\mathrm{Bi}_{2} \mathrm{WO}_{6}$ 和(c) $\mathrm{Bi}_{2} \mathrm{WO}_{6} / \mathrm{CNOs}$ 的 SEM 照 片以及(d) XRD 图谱

Fig. 1 SEM images of (a) CNOs, (b) $\mathrm{Bi}_{2} \mathrm{WO}_{6}$ and (c) $\mathrm{Bi}_{2} \mathrm{WO}_{6}$ CNOs samples; (d) XRD patterns of $\mathrm{CNOs}, \mathrm{Bi}_{2} \mathrm{WO}_{6}$ and $\mathrm{Bi}_{2} \mathrm{WO}_{6} / \mathrm{CNOs}$ samples

$\mathrm{Bi}_{2} \mathrm{WO}_{6}$ 和 $\mathrm{Bi}_{2} \mathrm{WO}_{6} / \mathrm{CNO}$ 的 XRD 图谱, $\mathrm{Bi}_{2} \mathrm{WO}_{6} /$ $\mathrm{CNOs}$ 在 $26.6^{\circ}$ 有一个明显的衍射峰, 这对应于 $(002)$ 石墨晶面，表明 CNOs 已经复合到 $\mathrm{Bi}_{2} \mathrm{WO}_{6}$ 中; 此外, 在 CNOs 的 XRD 图谱中, 四个分布在 $43.6^{\circ}, 51.3^{\circ}$, $75.1^{\circ}$ 和 $77.2^{\circ}$ 处的小峰为 $\mathrm{Fe}-\mathrm{Ni}$ 合金, 这是由于通过 CVD 法生成的 CNOs 中, 一部分会带有少量 $\mathrm{Fe}-\mathrm{Ni}$ 核 ${ }^{[21]} ; \mathrm{Bi}_{2} \mathrm{WO}_{6} / \mathrm{CNOs}$ 与 $\mathrm{Bi}_{2} \mathrm{WO}_{6}$ (JCPDS No. 39-0256) 表现出相似的晶相，四个衍射峰分别出现在 $28.47^{\circ}$ 、 $32.88^{\circ} 、 47.23^{\circ}$ 和 $56.01^{\circ}$ 处, 对应(131)、(020)、(220) 和(133)晶面, 表明 $\mathrm{Bi}_{2} \mathrm{WO}_{6} / \mathrm{CNOs}$ 仍然保持有纯相铇 酸铋的结构。

\section{2 电化学性能分析}

为了对材料的电容性能进行表征，材料在不同 扫描速度下的循环伏安曲线 $(\mathrm{CV})$ 如图 2 所示, $\mathrm{Bi}_{2} \mathrm{WO}_{6}$ 与 $\mathrm{Bi}_{2} \mathrm{WO}_{6} / \mathrm{CNOs}$ 都有一个分布在 $-0.9 \mathrm{~V}$ 附 近的较为尖锐的还原峰 $(\mathrm{R})$ 和一个分布在 $-0.6 \mathrm{~V}$ 附 近的氧化峰 $(O)$, 而且随着扫描速度的增大, 氧化还 原电位发生了略微的偏移, 表明样品均具有良好的 电容性和可逆的氧化还原性。根据 Vivier 等 ${ }^{[22]}$ 提出 的机理, 这些氧化还原峰对应于 $\mathrm{Bi}(\mathrm{III})$ 到 $\mathrm{Bi}(0)$ 的氧 化还原反应。

在还原过程中, 发生了如下反应:

$$
\begin{aligned}
& \mathrm{BiO}_{2}^{-}+\mathrm{e}^{-} \rightarrow \mathrm{BiO}_{2}^{2-} \\
& 2 \mathrm{H}_{2} \mathrm{O}+3 \mathrm{BiO}_{2}^{2-} \stackrel{\text { Disproportionation }}{\longleftrightarrow} \\
& 2 \mathrm{BiO}_{2}^{2-}+4 \mathrm{OH}^{-}+\mathrm{Bi}^{(0)}
\end{aligned}
$$
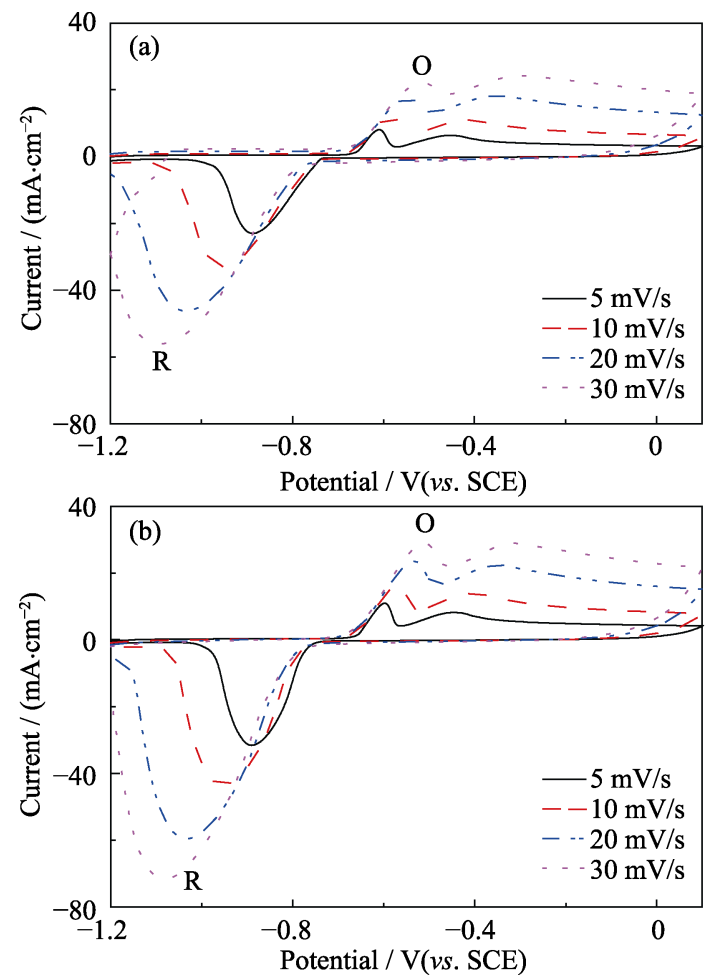

图 2 不同扫描速率下 (a) $\mathrm{Bi}_{2} \mathrm{WO}_{6}$ 和(b) $\mathrm{Bi}_{2} \mathrm{WO}_{6} / \mathrm{CNOs}$ 的 $\mathrm{CV}$ 曲线

Fig. $2 \mathrm{CV}$ curves of (a) $\mathrm{Bi}_{2} \mathrm{WO}_{6}$ and (b) $\mathrm{Bi}_{2} \mathrm{WO}_{6} / \mathrm{CNO}$ samples at various scan rates

$$
\mathrm{Bi}^{(0)} \rightarrow \mathrm{Bi}_{(\text {metal })}
$$

在氧化过程中, 发生了如下反应:

$$
\begin{gathered}
\mathrm{Bii}_{\text {(metal) }} \rightarrow \mathrm{Bi}^{+}+\mathrm{e}^{-} \\
\stackrel{\text { Disproportionation }}{\longrightarrow} \mathrm{Bi}^{3+}+2 \mathrm{Bi}_{(\text {metal })} \\
3 \mathrm{OH}^{-}+\mathrm{Bi}^{3+} \rightarrow \mathrm{Bi}(\mathrm{OH})_{3} \\
\mathrm{Bi}(\mathrm{OH})_{3} \rightarrow \mathrm{BiOOH}+\mathrm{H}_{2} \mathrm{O}
\end{gathered}
$$

通过 $\mathrm{CV}$ 曲线可以发现，在相同扫描速度下 $\mathrm{Bi}_{2} \mathrm{WO}_{6} / \mathrm{CNOs}$ 的积分面积略大于 $\mathrm{Bi}_{2} \mathrm{WO}_{6}$, 根据式(8) 可知, 材料的比电容与 $\mathrm{CV}$ 曲线的积分面积成正比

$$
C_{\mathrm{s}}=\frac{\int I \mathrm{~d} V}{2 \times \Delta V \times m \times \vartheta}
$$

其中, $C_{\mathrm{s}}$ 是循环伏安下的比电容 $\left(\mathrm{F} \cdot \mathrm{g}^{-1}\right), \int I \mathrm{~d} V$ 表示 $\mathrm{CV}$ 曲线的积分面积, $\Delta V$ 是电压窗口, $m$ 是活性物 质的质量， $\vartheta$ 是扫描速率。因而，当扫描速率为 $30 \mathrm{mV} \cdot \mathrm{s}^{-1}$ 时, $\mathrm{Bi}_{2} \mathrm{WO}_{6}$ 和 $\mathrm{Bi}_{2} \mathrm{WO}_{6} / \mathrm{CNOs}$ 的比电容分 别为 $237 、 364 \mathrm{~F} \cdot \mathrm{g}^{-1}$, 随着扫描速率的加快, 二者的 比电容均减小。此外, CNOs 在 $30 \mathrm{mV} \cdot \mathrm{s}^{-1}$ 的扫描速率 下, 比电容为 $4.82 \mathrm{~F} \cdot \mathrm{g}^{-1}$, 故而复合材料比电容的提 高不是由于 CNOs 的高比电容值, 这也佐证了 CNOs 本身的性质是提高复合材料电容性的关键。

图 3 是两种材料的充放电曲线, 二者曲线走势 

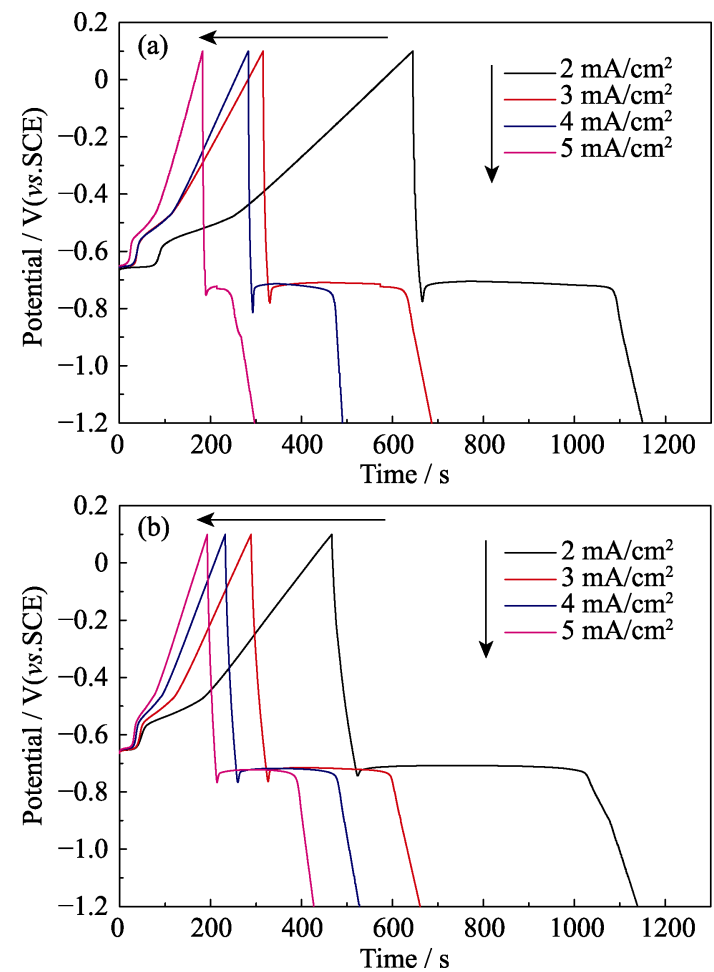

图 $3 \mathrm{Bi}_{2} \mathrm{WO}_{6}(\mathrm{a})$ 和 $\mathrm{Bi}_{2} \mathrm{WO}_{6} / \mathrm{CNO}$ (b) 的充放电曲线

Fig. 3 Charge-discharge curves of $\mathrm{Bi}_{2} \mathrm{WO}_{6}(\mathrm{a})$ and $\mathrm{Bi}_{2} \mathrm{WO}_{6} /$ CNOs (b) samples at various current density

相似, 表现出明显的赝电容特性。在放电过程中, 曲 线经历了三个阶段，首先，电位突然下降，这可归 因于溶液电阻; 随着氧化还原反应进行产生了电位 平台, 这与图 2 中 $\mathrm{CV}$ 的结果一致; 最终离子浓度极 化引起了电位下降, 完成放电。材料的比电容可以 通过下列公式 ${ }^{[23]}$ 进行计算:

$$
\begin{gathered}
E=\frac{C_{\mathrm{s}} V^{2}}{2} \\
C_{\mathrm{s}}=\frac{2 E}{m \cdot V_{2}^{2}-V_{1}^{2}}
\end{gathered}
$$

其中, $C_{\mathrm{s}}$ 是循环伏安下的比电容 $\left(\mathrm{F} \cdot \mathrm{g}^{-1}\right), V$ 是电压窗 口, $E$ 是能量密度, $m$ 是活性材料的质量, $V_{2}$ 是最大 电压 $(0.1 \mathrm{~V}), V_{1}$ 是最小电压 $(-1.2 \mathrm{~V})$ 。当电流密度为 $2 \mathrm{~mA} \cdot \mathrm{cm}^{-2}$ 时, $\mathrm{Bi}_{2} \mathrm{WO}_{6}$ 和 $\mathrm{Bi}_{2} \mathrm{WO}_{6} / \mathrm{CNOs}$ 的比电容分 别是 218 和 $328 \mathrm{~F} \cdot \mathrm{g}^{-1}$; 当电流密度增加到 $5 \mathrm{~mA} \cdot \mathrm{cm}^{-2}$ 时, 二者的比电容降低到 76 和 $173 \mathrm{~F} \cdot \mathrm{g}^{-1}$, 这是由于 随着电流密度的增加, 离子的电阻率也同时增加, 电荷扩散到活性中心的数量减少, 导致氧化还原反 应速率降低 ${ }^{[24]}$ 。因而, 可以推测 $\mathrm{Bi}_{2} \mathrm{WO}_{6} / \mathrm{CNOs}$ 比电 容的增加是由于 CNOs 的加入使材料的电阻率降低, 加速了电子的传输, 进而使活性物质的氧化还原速 率加快; 此外, 纯相 $\mathrm{Bi}_{2} \mathrm{WO}_{6} 、 \mathrm{CNOs}$ 和 $\mathrm{Bi}_{2} \mathrm{WO}_{6} / \mathrm{CNO}$ 的比表面积分别为 $106 、 14$ 和 $71 \mathrm{~m}^{2} \cdot \mathrm{g}^{-1}$ 。一般来说, 比 表面积越大, 活性物质的比容量越大, 由此推测, 虽 然 $\mathrm{Bi}_{2} \mathrm{WO}_{6} / \mathrm{CNOs}$ 的比表面积缩小, 但是 CNOs 的加
入可以增加纳米材料的导电性, 从而使活性物质 $\mathrm{Bi}_{2} \mathrm{WO}_{6}$ 的有效利用率提高, 比容量增加。

为了进一步研究两种材料的循环稳定性, 图 4 为材料在电流密度为 $5 \mathrm{~mA} \cdot \mathrm{cm}^{-2}$ 的条件下循环 300 次的性能图。 $\mathrm{Bi}_{2} \mathrm{WO}_{6}$ 和 $\mathrm{Bi}_{2} \mathrm{WO}_{6} / \mathrm{CNO}$ 的初始比电 容分别为 76 和 $173 \mathrm{~F} \cdot \mathrm{g}^{-1}$, 经过 300 次循环后变为 49 和 $149 \mathrm{~F} \cdot \mathrm{g}^{-1}$, 比电容的保持率分别为 $64 \%$ 和 $86 \%$, 由此可见, CNOs 的加入可以提高 $\mathrm{Bi}_{2} \mathrm{WO}_{6}$ 的 稳定性。

使用正弦电压脉冲, 在 $0.01 \mathrm{~Hz} 100 \mathrm{kHz}$ 的频 率范围内对材料进行交流阻抗测试。图 5 为 $\mathrm{Bi}_{2} \mathrm{WO}_{6}$ 和 $\mathrm{Bi}_{2} \mathrm{WO}_{6} / \mathrm{CNOs}$ 的 Nyquist 图谱, 插图为放大的 高频区域。Nyquist 图谱的高频区是一个半圆，这与 电极一电解质界面的电荷转移电阻相关, 半圆越小, 表明材料的电荷转移电阻越小; 低频区是一条直 线, 是由电解质离子进入或离开电极材料引起的, 坡度越陡的材料扩散阻力越低, 越接近理想的电容 器 ${ }^{[25]}$ 。Nyquist 图谱和实轴的第一个交点反映的是材 料在电解液中的传质内阻, 由图中可知, $\mathrm{Bi}_{2} \mathrm{WO}_{6}$ 和

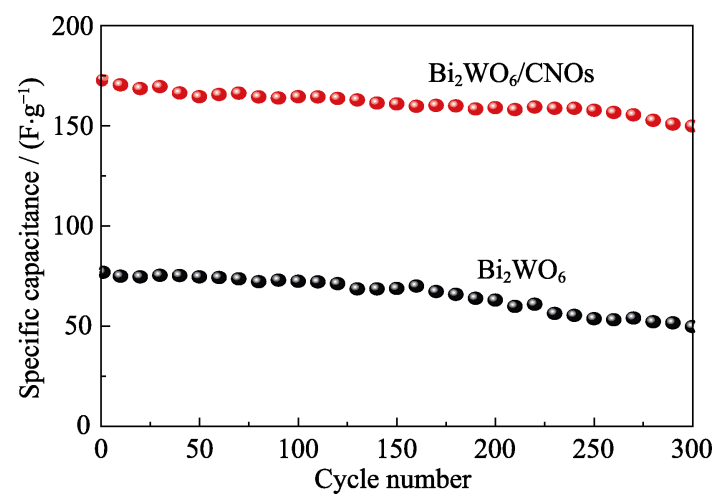

图 $4 \mathrm{Bi}_{2} \mathrm{WO}_{6}$ 和 $\mathrm{Bi}_{2} \mathrm{WO}_{6} / \mathrm{CNO}$ 的充放电循环性能图

Fig. 4 Capacitance retention of $\mathrm{Bi}_{2} \mathrm{WO}_{6}$ and $\mathrm{Bi}_{2} \mathrm{WO}_{6} / \mathrm{CNOs}$ samples as function of charge-discharge cycling number at a constant current density of $5 \mathrm{~mA} \cdot \mathrm{cm}^{-2}$

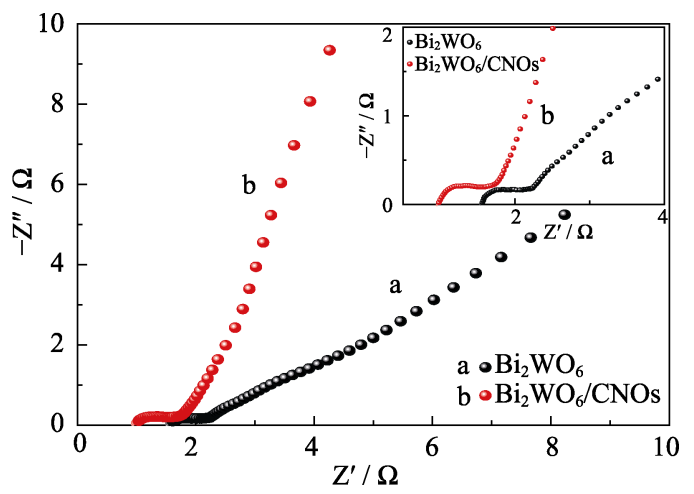

图 $5 \mathrm{Bi}_{2} \mathrm{WO}_{6}$ 和 $\mathrm{Bi}_{2} \mathrm{WO}_{6} / \mathrm{CNOs}$ 的 Nyquist 图谱, 插图为放 大的高频区域

Fig. 5 Nyquist plots of $\mathrm{Bi}_{2} \mathrm{WO}_{6}$ and $\mathrm{Bi}_{2} \mathrm{WO}_{6} / \mathrm{CNO}$ samples with inset showing the enlarged high-frequency region 
$\mathrm{Bi}_{2} \mathrm{WO}_{6} / \mathrm{CNOs}$ 的溶液内阻分别为 1.51 和 $0.98 \Omega$; 高频 区半圆的直径代表电荷转移电阻 ${ }^{[26]}$, 分别为 0.84 和 $0.72 \Omega$, 此外, 与纯相 $\mathrm{Bi}_{2} \mathrm{WO}_{6}$ 相比, $\mathrm{Bi}_{2} \mathrm{WO}_{6} / \mathrm{CNOs}$ 在 低频区的直线更接近于 $90^{\circ}$, 表明 CNOs 的加入明显 提高了 $\mathrm{Bi}_{2} \mathrm{WO}_{6}$ 的电容性能。

\section{3 结论}

通过水热法合成了 $\mathrm{Bi}_{2} \mathrm{WO}_{6}$ 和 $\mathrm{Bi}_{2} \mathrm{WO}_{6} / \mathrm{CNOs}$ 纳 米材料, 并通过电化学工作站对两种材料进行了循 环伏安、充放电和交流阻抗测试。可以看出, 两种 材料都表现出良好的赝电容特性, 对提高电容器的 电容性能起到了一定的作用。通过对两种材料进行 对比发现, 在以 $1 \mathrm{~mol} \cdot \mathrm{L}^{-1} \mathrm{KOH}$ 水溶液为电解液, 电 流密度为 $2 \mathrm{~mA} \cdot \mathrm{cm}^{-2}$ 的条件下, $\mathrm{Bi}_{2} \mathrm{WO}_{6}$ 和 $\mathrm{Bi}_{2} \mathrm{WO}_{6} /$ CNOs 的比电容分别是 218 和 $328 \mathrm{~F} \cdot \mathrm{g}^{-1}$; 在电流密 度为 $5 \mathrm{~mA} \cdot \mathrm{cm}^{-2}$ 的情况下, $\mathrm{Bi}_{2} \mathrm{WO}_{6}$ 和 $\mathrm{Bi}_{2} \mathrm{WO}_{6} / \mathrm{CNOs}$ 经过 300 次的充放电循环后, 比电容保持率分别为 $64 \%$ 和 $86 \%$, 由此可见, CNOs 的加入明显提高了 $\mathrm{Bi}_{2} \mathrm{WO}_{6}$ 的电化学性能。并且, 通过交流阻抗图可以 得出 $\mathrm{Bi}_{2} \mathrm{WO}_{6} / \mathrm{CNOs}$ 的内阻小于 $\mathrm{Bi}_{2} \mathrm{WO}_{6}$, 证明 $\mathrm{CNOs}$ 的加入改变了 $\mathrm{Bi}_{2} \mathrm{WO}_{6}$ 的电阻率, 能够加快电 子在电极和电解液中的传输, 并为电子提供相对短 的扩散路径, 从而加快材料的氧化还原反应速率, 增加噟电容的比容量。

\section{参考文献:}

[1] JABEEN N, HUSSAIN A, XIA Q Y, et al. High-performance $2.6 \mathrm{~V}$ aqueous asymmetric supercapacitors based on in situ formed $\mathrm{Na}_{0.5} \mathrm{MnO}_{2}$ nanosheet assembled nanowall arrays. Adv. Mater., 2017, 29 (32): 1700804-1-9.

[2] XIE Y X, LU L S, TANG Y, et al. Hierarchically nanostructured carbon fiber-nickel-carbon nanotubes for high-performance supercapacitor electrodes. Mater. Lett., 2017, 186: 70-73.

[3] XIAO Y H, SU D C, WANG X Z, et al. Layered double hydroxides with larger interlayer distance for enhanced pseudocapacitance. Sci. China Mater., 2017, 61(2): 263-272.

[4] CHEN S M, YANG G, JIA Y, et al. Three-dimensional $\mathrm{NiCo}_{2} \mathrm{O}_{4} @ \mathrm{NiWO}_{4}$ core-shell nanowire arrays for high performance supercapacitors. J. Mater. Chem. A, 2017, 5(3): 1028-1034.

[5] YAN H, BAI J, LIAO M, et al. One-step synthesis of $\mathrm{Co}_{3} \mathrm{O}_{4} /$ graphene aerogels and their all-solid-state asymmetric supercapacitor. Eur. J. Inorg. Chem., 2017, 2017(8): 1143-1152.

[6] YIN S, WU Y Q, XIONG Q Q, et al. Flower-like NiO with hierarchical and mesoporous structure for supercapacitor. Chemelectro., 2017, 4(10): 2563-2570.

[7] NITHIYANANTHAM U, EDE S R, ANANTHARAJ S, et al. Self-assembled $\mathrm{NiWO}_{4}$ nanoparticles into chain-like aggregates on DNA scaffold with pronounced catalytic and supercapacitor activities. Cryst. Growth Des., 2015, 15(2): 673-686.

[8] GHASEMI S, HOSSEINI S R, BOORE-TALARI O. Sonochemical assisted synthesis $\mathrm{MnO}_{2} / \mathrm{RGO}$ nanohybrid as effective electrode material for supercapacitor. Ultrason. Sonochem., 2018, 40(Pt A): 675-685.
[9] LI J S, LU W B, YAN Y S, et al. High performance solid-state flexible supercapacitor based on $\mathrm{Fe}_{3} \mathrm{O}_{4} /$ carbon nanotube/polyaniline ternary films. J. Mater. Chem. A, 2017, 5(22): 11271-11277.

[10] MARUTHAMANI D, VADIVEL S, KUMARAVEL M, et al. Fine cutting edge shaped $\mathrm{Bi}_{2} \mathrm{O}_{3}$ rods/reduced graphene oxide (RGO) composite for supercapacitor and visible-light photocatalytic applications. J. Colloid Interf. Sci., 2017, 498: 449-459.

[11] NITHYA V D, HANITHA B, SURENDRAN S, et al. Effect of pH on the sonochemical synthesis of $\mathrm{BiPO}_{4}$ nanostructures and its electrochemical properties for pseudocapacitors. Ultrason. Sonoche., 2015, 22: 300-310.

[12] CHOI D, JAMPANI P H, JAYAKODY J R P, et al. Synthesis, surface chemistry and pseudocapacitance mechanisms of VN nanocrystals derived by a simple two-step halide approach. Mater. Sci. Eng. B, 2018, 230: 8-19.

[13] NITHYA V D, SELVAN R K, KALPANA D, et al. Synthesis of $\mathrm{Bi}_{2} \mathrm{WO}_{6}$ nanoparticles and its electrochemical properties in different electrolytes for pseudocapacitor electrodes. Electrochim. Acta, 2013, 109(6): 720-731.

[14] HAI Z, AKBARI M K, XUE C, et al. Atomically-thin $\mathrm{WO}_{3} / \mathrm{TiO}_{2}$ heterojunction for supercapacitor electrodes developed by atomic layer deposition. Comp. Comm., 2017, 5: 31-35.

[15] AMBADE R B, AMBADE S B, SHRESTHA N K, et al. Controlled growth of polythiophene nanofibers in $\mathrm{TiO}_{2}$ nanotube arrays for supercapacitor applications. J. Mater. Chem. A, 2017, 5(1): 172-180.

[16] NITHYA V D, SELVAN R K, VASYLECHKO L, et al. Effect of carbon coating on the electrochemical properties of $\mathrm{Bi}_{2} \mathrm{WO}_{6}$ nanoparticles by PVP-assisted sonochemical method. J. App. Electrochem., 2015, 45(5): 473-485.

[17] JIAO Y, LIU Y, YIN B S, et al. Hybrid $\alpha-\mathrm{Fe}_{2} \mathrm{O}_{3} @ \mathrm{NiO}$ heterostructures for flexible and high performance supercapacitor electrodes and visible light driven photocatalysts. Nano Energy, 2014, 10: 90-98.

[18] LIU M X, ZHAO F L, ZHU D Z, et al. Ultramicroporous carbon nanoparticles derived from metal-organic framework nanoparticles for high-performance supercapacitors. Mater. Chem. Phy., 2018, 211: $234-241$

[19] LU W J, HUANG S Z, MIAO L, et al. Synthesis of $\mathrm{MnO}_{2} / \mathrm{N}$-doped ultramicroporous carbon nanospheres for high-performance supercapacitor electrodes. Chinese Chem. Lett., 2017, 28(6): 1324-1329.

[20] IIJIMA S. Direct observation of the tetrahedral bonding in graphitized carbon black by high resolution electron microscopy. $J$. Cryst. Growth, 1980, 50(3): 675-683.

[21] ZHANG W K, FU J J, CHANG J, et al. Fabrication and purification of carbon nano onions. New Carbon Mater., 2014, 29(5): 398403.

[22] VIVIER V, MEZAILLE S, WU B L, et al. Electrochemical study of $\mathrm{Bi}_{2} \mathrm{O}_{3}$ and $\mathrm{Bi}_{2} \mathrm{O}_{2} \mathrm{CO}_{3}$ by means of a cavity microelectrode $\mathrm{I}$. Observed phenomena and direct analysis of results. J. Electrochem. Soc., 2000, 147(11): 4252-4262.

[23] MAI L Q, MINHASKHAN A, TIAN X C, et al. Synergistic interaction between redox-active electrolyte and binder-free functionalized carbon for ultrahigh supercapacitor performance. Nat. Commun., 2013, 4(1): 2923-2931.

[24] AGHAZADEH M, GOLIKAND A N, GHAEMI M. Synthesis, characterization, and electrochemical properties of ultrafine $\beta$ $\mathrm{Ni}(\mathrm{OH})_{2}$ nanoparticles. Int. J. Hydrogen. Energ., 2011, 36(14): 8674-8679.

[25] LI F H, SONG J F, YANG H F, et al. One-step synthesis of graphene $/ \mathrm{SnO}_{2}$ nanocomposites and its application in electrochemical supercapacitors. Nanotechnology, 2009, 20(45): 455602 $1-6$.

[26] SUN L, TIAN C G, LI M T, et al. From coconut shell to porous graphene-like nanosheets for high-power supercapacitors. J. Mater. Chem. A, 2013, 1(21): 6462-6470. 